\title{
Cambio de imagen facial en base a rinoseptoplastía y cirugía ortognática: Resultados preliminares
}

\section{Facial makeover based in rhinoplasty and orthognathic surgery. Preliminary results}

\author{
Sergio Olate $\mathbf{M}^{1,2}$, Miguel Henríquez $\mathrm{A}^{3,4}$, Ignacio Huenchullán $\mathrm{C}^{5}$, Alejandro Unibazo $\mathrm{Z}^{1,2}$, Juan Pablo \\ Alister $\mathrm{H}^{1,6}$, Francisca Uribe $\mathrm{F}^{1,6}$.
}

\begin{abstract}
RESUMEN
Introducción: La cirugía ortognática y la rinoplastía son procedimientos consolidados con altas tasas de éxito. La sinergia entre ambos procedimientos ha sido presentada en diferentes investigaciones pero aún no es un procedimiento estándar para pacientes con deformidades faciales.

Objetivo: Analizar una serie de casos tratados con cirugía ortognática y rinoplastía y discutir las opciones y fundamentos de la técnica combinada.

Material y método: 14 pacientes operados de forma consecutiva fueron incluidos en el estudio. Se identificaron sujetos según el tipo de deformidad facial, el tipo de deformidad nasal y los tipos de cirugías realizadas. El seguimiento mínimo de cada sujeto fue de 12 meses.

Resultados: El 50\% de los sujetos presentaba deformidad facial de clase III, seguido de deformidades de clase II y I. Las deformidades nasales, de la punta y del puente nasal fueron más prevalentes, siendo el ancho nasal también una deformidad común. En la etapa intraoperatoria, después de realizar la cirugía ortognática, fue necesario realizar un análisis nuevo respecto de la morfología nasal debido a los cambios que generaba el movimiento maxilar en la condición nasal.

Conclusión: La cirugía combinada es sinérgica y viable de ser realizada con bajo número de complicaciones; protocolos y nuevos criterios de análisis son necesarios para obtener mayor predictibilidad en los resultados estéticos.
\end{abstract}

Palabras clave: Cirugía ortognática, rinoplastía, cirugía facial.

\footnotetext{
ABSTRACT

Introduction: Orthognathic surgery and rhinoplasty are procedures with high success rate. The synergy between both surgeries has been showed in the past by resear-

Departamento de Cirugía Oral, Facial y Maxilofacial, Facultad de Odontología, Universidad de La Frontera, Temuco, Chile.

2 Servicio de Cirugía Maxilofacial, Hospital Abraham González Peña, Lautaro, Chile.

3 Servicio de Otorrinolaringología, Clínica Alemana de Temuco, Temuco, Chile.

4 Departamento de Especialidades Médicas, Facultad de Medicina, Universidad de La Frontera, Temuco, Chile.

5 Servicio de Anestesiología, Clínica Alemana de Temuco, Temuco, Chile.

6 Servicio de Cirugía Maxilofacial, Hospital Hernán Henríquez Aravena, Temuco, Chile.

*Los autores declaran no tener conflictos de interés.

Recibido el 9 de mayo, 2018. Aceptado el 27 de junio, 2018.
} 
ches but still they are not standardized procedures for the treatment of patients with facial deformities.

Aim: To analyze a case series treated with orthognathic surgery and rinoplasty in the same surgical time, discussing surgical options and techniques in the combined procedure.

Material and method: 14 patients were treated consecutively and were included in this research. Subjects were selected by facial deformity, nasal deformity and type of surgery. Minimal follow up was for 12 months to observe results.

Results: $50 \%$ of subjects showed class III facial deformity, follow by class II and class I facial deformity. In nasal deformities, tip and nasal bridge were more prevalent, being the nasal width a common deformity; in the intraoperative time, after orthognathic surgery, it was necessary to make a new analysis and approach to nasal morphology because the new conditions related to movement of the maxilla.

Conclusion: Orthognathic and nasal surgery are good complement and it is possible to do with few complications; surgical protocol and criteria for the analysis are necessaries to obtain more predictability in the esthetic results.

Key words: Orthognathic surgery, rhinoseptoplasty, facial surgery.

\section{INTRODUCCIÓN}

La deformidad facial es una enfermedad que genera múltiples alteraciones y secuelas, comprometiendo función a diferentes niveles junto a una importante implicancia en la estética facial ${ }^{1}$. Las alteraciones de la estética facial pueden variar en magnitud y se deben incluir dentro de los objetivos de tratamiento, de la misma forma en que se integran otras condicionantes funcionales ${ }^{2,3}$.

Cuando existe una posición incorrecta en la estructura máxilo-mandibular debido a deformidades, es habitual observar otras anomalías del tercio medio facial, donde la nariz tiene un rol fundamental; una posición más inferior 0 excesivamente adelantada de la maxila implica el mismo cambio en la posición de espina nasal anterior (ENA), determinando una anomalía de posición de la base nasal ${ }^{4}$.

En este sentido, investigaciones previas de nuestro grupo han demostrado que el $85 \%$ de los sujetos con deformidad esqueletal sagital de clase III presenta alteraciones en la posición del septum nasal y en toda la morfología nasal ${ }^{5}$, de forma que cualquier intervención ortognática a nivel de maxila va a generar nuevas condiciones en la función y morfología nasal. En este sentido, Dantas y cols $^{6}$. indicaron que la cirugía maxilar que lleva a su reposición superior y anterior genera $85 \%$ de movilización de la punta nasal hacia anterior, 80\% de rotación de la punta nasal y ensanchamiento de la base alar en el 95\%, causando importantes cambios estéticos y morfológicos en esta área. En casos de deformidades maxilares y nasales, la cirugía exclusiva solo ortognática o solo rinoplastía son insuficientes, instalando nuevas limitaciones en futuras correcciones morfofuncionales (Figuras 1 y 2 ).

La rinoplastía en conjunto con la cirugía ortognática ha sido presentada en otras publicaciones $^{7}$, demostrando la eficiencia y eficacia del procedimiento. Uno de los primeros estudios publicados en este ámbito fue el de Waite y cols ${ }^{8}$, quienes operaron 22 pacientes determinando un protocolo para el manejo de la intubación y la secuencia quirúrgica para obtener resultados estéticos y funcionales; más tarde, Costa y cols $^{9}$, publicaron una secuencia de 13 pacientes operados con osteotomía Le Fort I y rinoplastía evidenciando algunos puntos a ser mejorados, pero con éxito en la evaluación final. La rinoseptoplastía junto a la mentoplastía están dentro de las 5 cirugías estéticas más desarrolladas en el mundo ${ }^{10}$, por lo que su impacto en la estética facial debe ser valoradas correctamente, de forma que combinar una cirugía nasal y una cirugía máxilo-mandibular tiene sinergia en resultados funcionales y estéticos. 


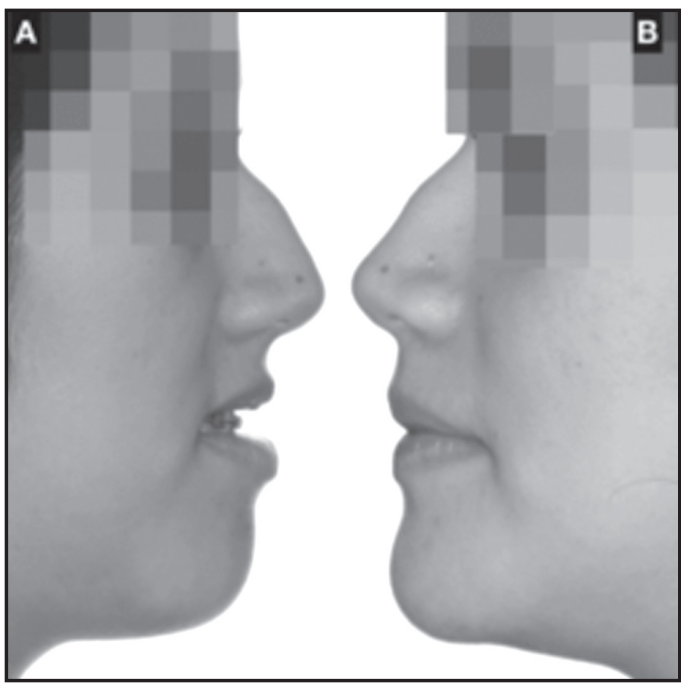

Figura 1. Paciente operada con cirugía ortognática bimaxilar y genioplastía. Aún es posible observar la deformidad de dorso nasal que podría ser mejorada con una rinoplastía. (A: etapa prequirúrgica, B: 17 meses después de realizada la cirugía ortognática).

\section{OBJETIVO}

El objetivo de esta investigación es reportar el resultado obtenido en una secuencia de 14 pacientes operados de forma conjunta mediante cirugía ortognática y rinoplastía.

\section{MATERIAL Y MÉTODO}

Se revisó una serie de casos operados bajo el concepto de cambio de imagen facial que integra la cirugía combinada con técnicas de cirugía ortognática y de rinoseptoplastía entre los años 2015 y 2017. Se incluyeron sujetos de entre 18 y 45 años, de ambos sexos, que consultaron en el Departamento de Cirugía Oral, Facial y Maxilofacial de la Universidad de La Frontera, para corrección ortoquirúrgica debido a la presencia de deformidad facial; todos los sujetos firmaron un consentimiento para participar en la investigación. Fueron excluidos sujetos que presentaron malformaciones faciales, antecedentes de trauma maxilofacial

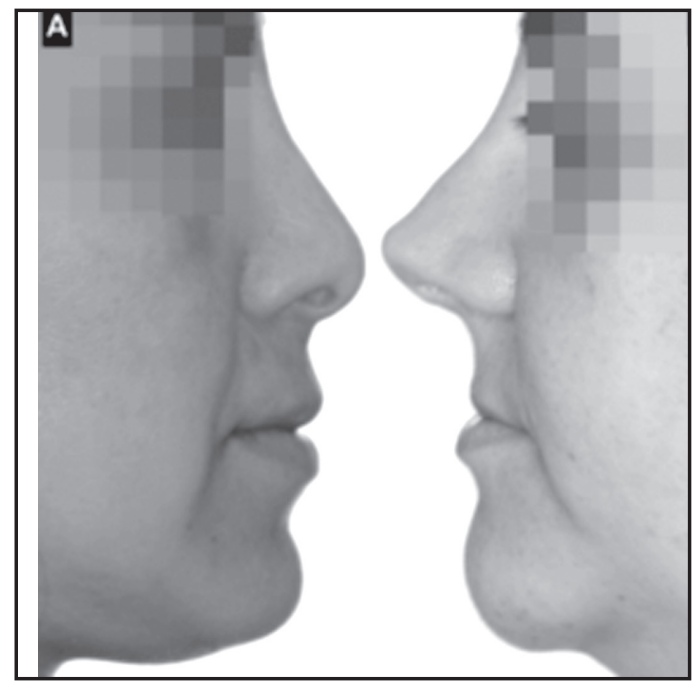

Figura 2. Rinoseptoplastía ejecutada en dos pacientes con deformidad facial de clase III. A: dorso nasal y ángulo nasolabial adecuado con deficiencia de la proyección sagital del tercio medio facial con aumento en proyección mandibular; B: excesiva rotación de la punta nasal, ángulo nasolabial abierto y deficiencia en el labio superior y región paranasal. En ambos casos es necesario realizar cirugía ortognática.

y antecedentes de patología maxilofacial que presentaran indicación de cirugías reconstructivas; también se excluyeron sujetos con asimetrías faciales asociadas a patología de la articulación témporo-mandibular como la hiperplasia 0 hipoplasia de cóndilo mandibular.

\section{Diagnóstico}

La deformidad facial que presentaban los sujetos incluidos fueron clasificados a nivel sagital (clase II y clase III), vertical (exceso vertical de maxila 0 deficiencia vertical de maxila) 0 a nivel transversal (compresión o exceso transversal máxilo mandibular); los diagnósticos fueron realizados mediante cefalometría $2 \mathrm{D}$ utilizando las indicaciones propuestas por McNamara y por Ricketts ${ }^{11} \mathrm{y}$ el análisis facial fue realizado según los parámetros propuestos por Epker ${ }^{12}$. La deformidad nasal fue analizada según sus aspectos y áreas anatómicas incluidas, registrando deformidades en el dorso nasal, área de la punta nasal (puntas caídas, rotadas, cuadradas, anchas, bífidas 0 
asimétricas o crura lateral excesivamente larga que da como resultado una mayor proyección de la punta), área alar y la columela (columela bífida, columela colgante y otras alteraciones columelares). La longitud y el ancho de la nariz y el ancho de la base alar (bases alares anchas 0 angostas.) también se registraron y analizaron para establecer las desviaciones nasales ${ }^{13}$. Gibas 0 deformidades en silla de montar se clasificaron como deformidades del puente nasal.

\section{Planificación}

Para la indicación y definición quirúrgica, se estableció un equipo de trabajo que incluía las áreas de otorrinolaringología, cirugía maxilofacial, anestesiología y ortodoncia. La planificación quirúrgica de la cirugía ortognática se realizó utilizando sistemas convencionales 2D 0 con sistema de planificación quirúrgica virtual según el año en que el paciente fue incorporado a tratamiento. La estructura de análisis se desarrolló según los movimientos necesarios para alcanzar función estable y armonía facial.

Luego de realizada la planificación de la cirugía ortognática, se realizó el estudio en base a Photoshop para la rinoseptoplastía que implica cambios importantes en relación a la rinoplastía convencional, ya que después de la cirugía ortognática se produce una ampliación de la base de los alares y cambios en toda la zona perioral, especialmente labio superior y comisura labial; además se puede presentar un aumento de la rotación de la punta nasal debido a cambios en la posición de la espina nasal anterior.

\section{Procedimiento quirúrgico}

\section{Cirugía ortognática}

En todos los casos de cirugía combinada con rinoseptoplastía, el inicio de la cirugía ortognática se realiza en la mandíbula mediante técnicas de rutina. La cirugía maxilar se realiza mediante un acceso vestibular y exposición de toda la zona de interés desarrollando la osteotomía Le Fort I de forma convencional. Con la maxila descendida se realiza un abordaje a la zona de septum y vómer, realizando la reducción vertical para que la nueva posición de la maxila no genere desvíos de septum y de la punta debido a la compresión que se puede generar cuando la maxila asciende a su nueva posición; la reducción del tabique es a nivel de la zona anterior principalmente, incluyendo el vómer en algunos casos; dependiendo del movimiento maxilar la realización inmediata de una turbinectomía por vía de la osteotomía de Le Fort I puede estar indicada.

Una vez fijada la maxila con osteosíntesis se procedió a realizar una captura de las bases alares mediante una sutura interlar transfixiante cuidando de mantener una relación próxima a la distancia intercantal en la etapa intraoperatoria. Posteriormente se realiza la fijación del septum con una sutura de nylon 3-0 a la zona de la espina nasal anterior a fin de disminuir la movilidad del septum para que en el tiempo de cirugía nasal exista mayor estabilidad para la manipulación quirúrgica. La sutura del acceso maxilar se realiza de forma convencional utilizando la técnica de V-Y para la reposición del labio superior. Toda esta intervención es realizada con hipotensión controlada.

\section{Cambio de intubación nasotraqueal a orotraqueal}

En el paso de cirugía ortognática hacia rinoplastía, la intubación del paciente cambia desde nasal hacia oral, donde el anestesiólogo utiliza maniobras convencionales considerando: (1) ventilación 100\% $0^{2}$, (2) retiro cuidadoso de packing orofaríngeo y aspiración, (3) utilización de sistema de imágenes de apoyo para intubación (videolaringoscopio), (4) introducción de nuevo tubo en boca, (5) bajo visualización directa de ambos tubos (nasal por retirar y nuevo tubo oral), se desinfla el cuff del tubo nasotraqueal, retirando el tubo nasal de las cuerdas para introducir el tubo oral con conductor, asegurando la vía aérea de forma rápida y segura y (6) fijación del nuevo tubo y posterior retiro total del tubo nasal.

\section{Rinoseptoplastía}

Inicialmente se realiza la septoplastía a través de un abordaje clásico, tanto por la experiencia del otorrinolaringólogo, asícomo para tener un segundo acceso que sirva para reposicionar un eventual cartílago sobrante, el cual evitaría un eventual efecto bandera del tabique y permitiría contar con material para eventuales reintervenciones. La 
rinoseptoplastía desarrollada en todos los casos fue de tipo abierta, abordando desde la punta nasal con incisión en $\mathrm{V}$ invertida. Para mantener la fortaleza de la punta nasal y para controlar transversalmente la base alar empleamos sutura PDS 3-0 en la base, pasando por los tejidos blandos y la ENA. En algunos casos hemos debido realizar resección de la base alar a nivel lateral, especialmente en casos de base alar ancha primaria o secundaria debido a cambios generados por la cirugía ortognática 0 por asimetrías a este nivel. La resección de la giba debe ser conservadora debido a los movimientos previos de la maxila, la que además de aumentar la punta nasal cefálicamente y avanzarla en sentido anterior permitirá que la deformidad dorsal sea menos prominente; la cantidad de reducción dorsal final, frecuentemente, es menor que la estimación preoperatoria, pudiendo correr el riesgo de un efecto de silla de montar. Las osteotomías laterales se realizan sin dificultad por el borde piriforme expuesto. En términos de manejo de dorso y punta, la técnica no cambia de procedimientos convencionales. En varios casos reforzamos la pirámide a través de la realización de injertos tipo spreader graft.

\section{RESULTADOS}

Se intervinieron 14 pacientes con la secuencia descrita. En todos ellos se ha mantenido un análisis extensivo y seguimiento por mínimo de 12 meses posquirúrgico, que está dentro de los tiempos estimados para el retiro de aparatos de ortodoncia. El tipo de deformidad más frecuente fue las de tipo III (Tabla 1), mientras que la deformidad nasal fue observada en diferentes niveles. En términos del tipo de cirugía (Tabla 2), existió mayor frecuencia de cirugías bimaxilares, donde la rinoplastía fue realizada frecuentemente con osteotomías.

En términos de tiempo quirúrgico, éste ha disminuido sustancialmente desde la primera cirugía combinada. El sangrado intraoperatorio es controlado mediante técnicas de hipotensión inducida, la cual es regulada durante el procedimiento en colaboración con infiltraciones de lidocaína con epinefrina para generar vasoconstricción en los sitios quirúrgicos de incisiones. En la fase de transición después del cambio de tubo y previo al inicio de la rinoseptoplastía, es necesario realizar una secuencia de análisis clínico que define medidas y formas debido a los cambios nasales generados por la ci-

Tabla 1. Descripción de las deformidades nasales observadas en 14 sujetos que presentaron deformidades esqueletales de clase I, II o III

\begin{tabular}{|lccc|}
\hline Deformidad & $\begin{array}{c}\text { Clase I esqueletal } \\
(\mathrm{n}=2)\end{array}$ & $\begin{array}{c}\text { Clase II esqueletal } \\
(\mathrm{n}=5)\end{array}$ & $\begin{array}{c}\text { Clase III esqueletal } \\
(\mathrm{n}=7)\end{array}$ \\
\hline Punta nasal & 1 & 0 & 7 \\
Puente nasal & 1 & 4 & 3 \\
Columela & 0 & 1 & 2 \\
Desviación nasal & 1 & 2 & 2 \\
Ancho nasal & 0 & 2 & 6 \\
\hline
\end{tabular}

Tabla 2. Descripción de las intervenciones quirúrgicas desarrolladas en los 14 pacientes operados con cirugía ortognática y rinoplastía

\begin{tabular}{|lccc|}
\hline Cirugía & $\begin{array}{c}\text { Clase I esqueletal } \\
(\mathrm{n}=2)\end{array}$ & $\begin{array}{c}\text { Clase II esqueletal } \\
(\mathrm{n}=5)\end{array}$ & $\begin{array}{c}\text { Clase III esqueletal } \\
(\mathrm{n}=7)\end{array}$ \\
\hline Cirugía maxilar & 2 & 3 & 7 \\
Cirugía mandibular & 2 & 5 & 6 \\
Cirugía de mentón & 2 & 4 & 5 \\
Cirugía nasal con osteotomía & 1 & 4 & 7 \\
Cirugía nasal sin osteotomía & 1 & 1 & 0 \\
\hline
\end{tabular}


rugía ortognática. En términos de complicaciones, en un caso se observó desvío de la punta nasal corregida con una segunda intervención después de 5 meses; un segundo caso presentó un orificio nasal de mayor tamaño al compararlo con el contralateral probablemente por resección asimétrica.

\section{DISCUSIÓN}

Las deformidades dentofacial y nasal presentan rutas semejantes en su génesis. En el desarrollo cráneo-facial, después de los 10 años de edad se produce un crecimiento importante de los tercios medio e inferior del rostro. Una función significativa en este crecimiento es comandado por el cóndilo mandibular, que entre los 10 y 15 años puede aumentar de 10 y $15 \mathrm{~mm}$ de altura, generando un explosivo cambio de posición de la mandíbula ${ }^{14}$. La maxila se integra con la mandíbula a través de la oclusión dentaria, la cual debe mantenerse en clase I (relación entre molares y caninos superiores e inferiores), respetando una relación aceptable entre maxilares ${ }^{15}$. Cerca del 30\% de las mal posiciones dentarias están asociadas con alteraciones del posicionamiento de maxilares, generando prognatismo, retrognatia y restricciones transversales, entre otras ${ }^{1}$; con ello, la base nasal está fuertemente comprometida, ya que la posición de la espina nasal anterior y el piso nasal responden a la posición del maxilar. Los huesos propios de la nariz, por otra parte, crecen en conjunto con toda la estructura nasofrontal y mantiene un crecimiento entre los 2 y 17 años de edad mediante remodelación que ocurre en los límites superior e inferior ${ }^{16}$. En este sentido, Sarnat ${ }^{17-19}$, mediante estudios experimentales, demostró que para obtener adecuado crecimiento maxilar y del paladar, la correcta posición del septum nasal y el vómer eran necesarias, lo cual está relacionado con el crecimiento de la estructura nasofrontal.

De un punto de vista puramente estético, la rinoplastía se integraba frecuentemente con los implantes aloplásticos de mentón para conseguir resultados de modificación facial con énfasis en el perfil del paciente ${ }^{10}$; en casos de deformidades faciales (aun siendo casos de baja severidad), la integración de cirugía correctiva máxilo mandibular con la rinoplastía tienen un papel funcional y estético, permitiendo resultados estables a largo plazo ${ }^{8}$. Tratamientos efectuados en pacientes con deformidades en base a rinoplastía y en ausencia de cirugía ortognática pueden traer complejas consecuencias en el tratamiento futuro funcional y estético de la deformidad facial (Figura 1); por otra parte, la cirugía ortognática exclusiva puede no solucionar algunas deformidades nasales asociadas (Figura 2).

En este análisis, es necesario recordar que cuando la maxila es más angosta que el tamaño necesario para la función conjunta con la mandíbula, es posible observar una apertura piriforme estrecha con la consecuente compresión de estructuras nasales; si la maxila es retraída, la base nasal tendrá limitado soporte y la ENA estará retrasada contribuyendo a un descenso de la punta nasal; si la maxila es prognática, la base nasal tendrá un efecto contrario y con la ENA más adelantada la nariz tendrá un rotación cefálica ${ }^{7}$. Con la maxila en una posición más inferior, acompañando la característica sonrisa gingival del exceso de crecimiento vertical del maxilar, la ENA se puede instalar en una posición más inferior y con ello la morfología nasal se observará alargada y estrecha, aumentando la deformidad del puente nasal y finalmente si la maxila se ubica en un patrón más superior, caracterizando la deficiencia de crecimiento vertical de maxila, la nariz se verá verticalmente pequeña, de base alar ancha y con punta descendida ${ }^{15}$. Dicho de otro modo, las condiciones de posición y forma de la maxila son fundamentales en el desarrollo estructural de la nariz y traen consecuencias funcionales y estéticas relevantes que deben ser incluidas en la planificación quirúrgica de pacientes candidatos a cirugía ortognática 0 a rinoseptoplastía. Por otra parte, cuando un paciente es sometido a una osteotomía de Le Fort I, se generará un cambio en la morfología nasal, donde las cirugías de avance maxilar aumentan el ancho interalar en al menos $2,5 \mathrm{~mm}^{3,20}$, motivo que justifica el uso de diferentes técnicas para optimizar la relación nasal y labial en la etapa posoperatoria de la cirugía ortognática.

La forma en que la estructura nasal responde a los movimientos maxilares no ha sido totalmente definida. La posición de nariz estudiada mediante en punto pronasal indicó un pobre acompañamiento de tejidos blandos al movimiento esqueletal siendo inferiores al $30 \%$ en dos estudios ${ }^{21,22}$; el 
estudio de Enacar y cols $^{23}$, sin embargo, mostró una relación próxima a 1:1 en análisis semejantes ${ }^{23}$. El área subnasal ha sido el área de mayor análisis, y aunque la variabilidad de resultados continúa presente, se observa que solo un estudio presenta relaciones de tejidos duros y blandos menores al $50 \%{ }^{24}$. Por otra parte, Altman y Oeltjen ${ }^{25}$, discutiendo el papel de la rinoplastía durante la cirugía bimaxilar, concluyeron la dificultad y definitivamente la incapacidad de predecir los cambios de la morfología nasal; también ellos indicaron que el solo acceso quirúrgico para la osteotomía de Le Fort I podría influenciar en el ensanchamiento de la basa nasal. Park y cols ${ }^{26}$, trabajando en base a tomografías de haz cónico indicaron cambios significativos de ensanchamiento en paciente bajo osteotomía bimaxilar, aun utilizando sutura de cincha labial superior y la sutura en forma de V-Y. Esta dificultad de análisis aumenta cuando Lim y cols ${ }^{27}$ presentaron movimientos de la punta nasal hacia anterior después de cirugías mandibulares exclusivas y cuando Jung y cols ${ }^{28}$ indicaron que el retroceso mandibular exclusivo influye en la posición de nariz y labio superior debido a que la musculatura perioral también estaría en remodelación.

Definitivamente, casos donde la rinoplastía presenta una clara indicación se caracterizan por presentar base alar ancha 0 amplia y escalón nasal moderado o prominente ${ }^{29}$. En este sentido, los pacientes que se benefician con esta doble intervención son (1) los pacientes que presentan una deformidad nasal previa a la cirugía y (2) los pacientes que desarrollan una deformidad como consecuencia de la nueva posición de la maxila y los efectos de la osteotomía Le Fort ${ }^{30}$.

De acuerdo con nuestras observaciones, la rinoplastía realizada después de la osteotomía de Le Fort I, presenta algunas particularidades que no están presentes en rinoplastías convencionales como: (1) presentar un septum nasal totalmente desinsertado en su posición inferior, (2) presentar una posición de la base nasal distinta a la observada en la etapa preoperatoria (generalmente más ancha) (3) presentar una nueva proyección anterior y una nueva estructura de relación en el puente nasal lo que implica la aplicación de técnicas que deben ser definidas de forma intraoperatoria. Los casos realizados bajo el protocolo presentado en esta investigación demostraron ser eficientes en su resultado estético y funcional (Figuras 3 y 4). La secuencia quirúrgica fue ordenada, precisa y con complicaciones semejantes a la observadas en procedimientos de rinoplastía exclusiva 0 de cirugía ortognática exclusiva.

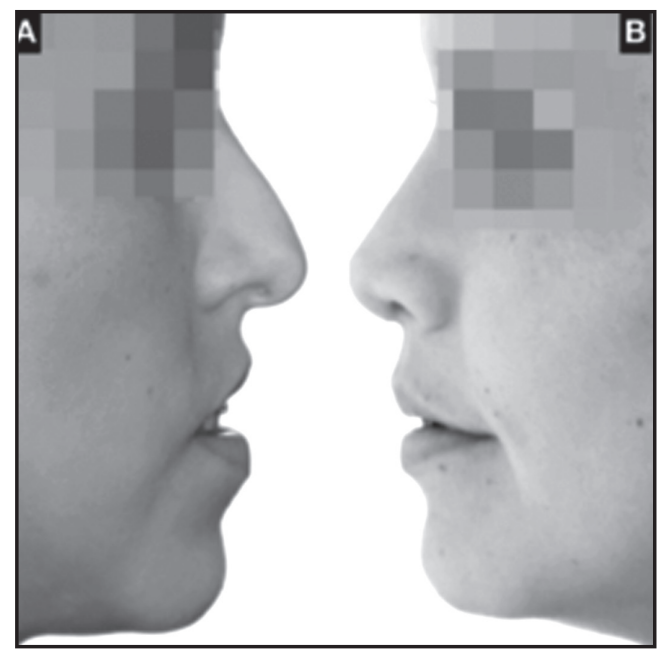

Figura 3. Paciente con deformidad facial de clase III y descenso de la punta nasal sometida a cirugía combinada. (A): etapa prequirúrgica, (B): 20 meses después de realizada la cirugía combinada. Se realizó avance maxilar de $6 \mathrm{~mm}$, retroceso mandibular y rotación de $3 \mathrm{~mm}$, ascenso de mentón de $4 \mathrm{~mm}$ y rinoplastía abierta con osteotomías.

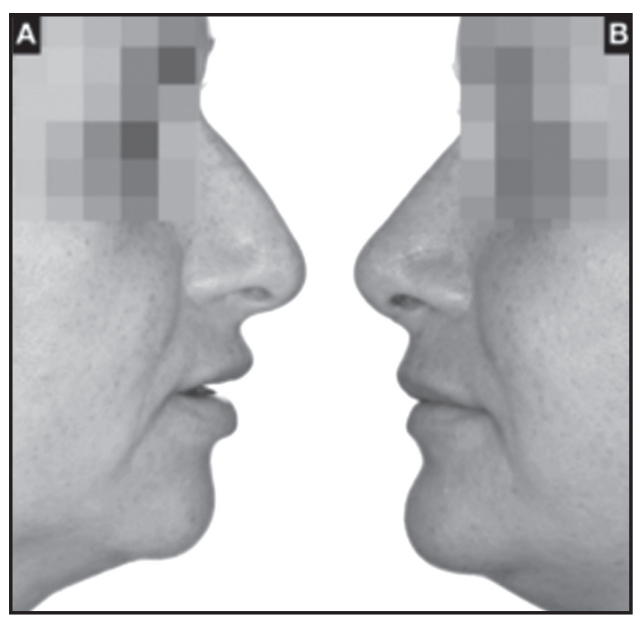

Figura 4. Paciente con deformidad facial de clase III y deformidad del puente nasal sometida a cirugía combinada. (A): etapa prequirúrgica, $(B)$ : 10 meses después de realizada la cirugía combinada. Se realizó avance de maxila de $5 \mathrm{~mm}$ y reposición de mentón con avance de $4 \mathrm{~mm}$ y ascenso de 3 $\mathrm{mm}$ para optimizar surco mento-labial. Se realizó rinoplastía abierta con osteotomías. 


\section{CONCLUSIÓN}

La cirugía ortognática y rinoseptoplastía pueden colaborar para optimizar los resultados estéticos

\section{BIBLIOGRAFÍA}

1. Joshi N, Hamdan A, Fakhouri W. Skeletal malocclusion: A developmental disorder with a life-long morbidity. J Clin Med Res 2014; 6: 399-408.

2. Figueroa J, Sanza C, Suazo N, Olate S. Parámetros de estética facial: Análisis descriptive de la clase facial y la sonrisa de sujetos jóvenes. Int J Med Surg Sci 2014; 1: 12-6.

3. Olate S, Zaror C, Mommaerts MY. A systematic review of soft-to-hard tissue ratios in orthognathic surgery. Part IV: 3D analysis - is there evidence? J Craniomaxillofac Surg 2017; 45: 1278-86.

4. Jeong HI, Lee HS, Jung YS, Park HS, Jung HD. Nasal soft tissue change following bimaxillary orthognathic surgery. J Craniofac Surf 2017; 28: e605-8.

5. Olate S, Cantín M, Muñoz M, Alister JP, Vásquez B, Chaves Netto H. Nasal deformity in subjects with class III facial deformities. Int J Morphol 2015; 33:1536-41.

6. Dantas, WR; da Silveira MM; do Egito Vasconcelos, $B C$ \& Porto, GG. Evaluation of the nasal shape after orthognathic surgery. Braz J Otorhinolaryngol 2015; 81:19-23.

7. Seah TE, Bellis H, Ilankovan V. orthognathic patients with nasal deformities: case for simultaneous orthognathic surgery and rhinoplasty. Br J Oral Maxillofac Surg 2012; 50 : 55-9.

8. Waite PD, Matukas VJ \& Sarver DM. Simultaneous rhinoplasty procedures in orthognathic surgery. Int J Oral Maxillofac Surg 1988; 17: 298-302.

9. Costa F, Robiony M, Salvo I, Toro C, Sembronio $S$ \& PoLITI M. Simultaneous functional endoscopic sinus surgery and esthetic rhinoplasty in orthognathic patients. J Oral Maxillofac Surg 2008, 66: 1370-7.

10. Fan K, Kawamoto HK, McCarthy JG, Bartlett SP, Mathews DC, Wolfe Sa, Tanna N, Vu MT, Bradley JP. Top five craniofacial techniques for training en el cambio de imagen facial; es necesario definir parámetros para mejorar la predictibilidad del procedimiento conjunto.

in plastic surgery residency. Plast Reconstr Surg 2012; 129: 477e-87e.

11. Guerrero M, Ocampo J, Olate S. Comparación entre las técnicas de Ricketts y McNamara para la determinación de la posición del maxilla y la mandibular en jóvenes del Ecuador. Int $J$ Morphol 2018; 36: 169-174.

12. Koury ME, EpKer BN. Maxillofacial esthetics: anthropometrics of the maxillofacial region. $J$ Oral Maxillofac Surg 1992; 50: 806-20.

13. Starck WJ, Epker BN. Cephalometric analysis of profile nasal esthetics. Part I. Method and normative data. Int J Adult Orthodon Orthognath Surg 1996; 11: 91-103.

14. Buschang $P$, Gandini L. Mandibular skeletal growth and modelling between 10 and 15 years of age. Eur J Orthod 2002; 24: 69-79.

15. Olate S, Chaves Netto HDM. Manipulación del plano oclusal en cirugia ortognática. Int $J$ Odontostomatol 2010; 4: 23-32.

16. Lestrell PE, Engstrom C, Chaconas SJ. A longitudinal study of the human nasal bone in Norma Lateralis: size and shape considerations. In: Dixon $A D$, Sarnat BG, Hoyte DAN, editors. Fundamentals of Bone Growth: Methodology and Applications. London: CRC Press. Inc.; 1991. pp. 547-64.

17. SaRnat BG. The face and jaws after surgical experimentation with the septovomeral region in growing and adult rabbits. Acta Otolaryngol Supp/ 1970; 268: 1-30.

18. Sarnat BG, WeXler MR. Longitudinal development of upper facial deformity after septal resection in growing rabbits. Br J Plast Surg 1969; 22 : 313-23.

19. Sarnat BG, Wexler MR. The snout after resection of nasal septum in adult rabbits. Arch Otolaryngol 1967; 86: 463-6.

20. Olate S, Zaror C, Blythe JN, Mommaerts MY. A systematic review of soft-to-hard tissue in orthognathic surgery. Part III: Double jaw surgery procedures. J Craniomaxillofac Surg 2016; 44: 1599-606. 
21. Conley RS, Boyd SB. Facial soft tissue changes following maxillomandibular advancement for treatment of obstructive sleep apnea. J Oral Maxillofac Surg 2007; 65: 1332-40.

22. Marsan G, Cura N, Emekli U. Soft and hard tissue changes after bimaxillary surgery in Turkish female class III patients. J Craniomaxillofac Surg 2009; 37: 8-17.

23. Enacar A, Taner T, Toroglu S. Analysis of soft tissue profile changes associated with mandibular setback and double jaw surgeries. Int J Adult Orthod Orthognath Surg 1999; 14: 27-35.

24. LiN S-S, KeRR WJ. Soft and hard tissue changes in class III patients treated by bimaxillary surgery. Eur J Orthod 1998; 20: 25-33.

25. Altman JI, Oeltuen JC. Nasal deformities associated with orthognathic surgery: analysis, prevention, and correction. J Craniofac Surg 2007; 18: 734-9.

26. Park SB, Yoon JK, Kim YI, Hwang DS, Cho $B H$, Son WS. The evaluation of the nasal morphologic changes after bimaxillary surgery in skeletal Class III malocclusion by using the superimposition of cone-beam computed tomography (CBCT) volumes. J CRANIOMAXILLOFAC SURG 2012; 40: e87-92.

27. Lim YK, Chu EH, Lee DY, Yang IH, Baek SH. Threedimensional evaluation of soft tissue change gradients after mandibular setback surgery in skeletal Class III malocclusion. Angle Orthod 2010; 80: 896-903.

28. Jung YJ, KIM MJ, BaEK SH. Hard and soft tissue changes after correction of mandibular prognathism and facial asymmetry by mandibular setback surgery: three-dimensional analysis using computerized tomography. Oral Surg Oral Med Oral Pathol Oral Radiol Endod 2009; 107: 763-71.

29. Güzel MZ, Saraç M, Arslan H, Nejat E, Nazan K. A new face by combined surgery for patients with complex dentofacial deformity. Aesth Plast Surg 2007; 31: 32-41.

30. Jones JK, Smith BR. Rationale for aesthetic surgery in conjunction with orthognathic surgery: before, during, and after orthognathic surgery. Oral Maxillo-fac Surg Clin North Am 1996; 8: 135-44.

\footnotetext{
Correspondencia: Sergio Olate

Departamento de Cirugía Oral, Facial y Maxilofacial Universidad de La Frontera. Claro Solar 115, Temuco, Chile

E mail: sergio.olate@ufrontera.cl
} 